\title{
SER OU NÃO TER, EIS A QUESTÃO: UM ESTUDO SOBRE MATERIALISMO E ESTILO DE VIDA
}

\section{TO BE OR NOT TO OWN, THAT IS THE QUESTION: A STUDY ON MATERIALISM AND LIFESTYLE}

Recebido: 30/01/2014

Aceite: 03/04/2014

Sofia Batista Ferraz ${ }^{1}$

Silvia Maria Dias Pedro Rebouças ${ }^{2}$

Giselle Cavalcante Queiroz ${ }^{3}$

Davi de Castro Rocha ${ }^{4}$

\section{RESUMO}

O papel da cultura de consumo tem sido progressivamente questionado enquanto vetor de felicidade e bem-estar à sociedade. A ideia de refuto aos códigos mercadológicos impostos à comunidade é enfatizado em prol da adoção de valores de equilíbrio interno e com o ambiente. Assim, mediante uma pesquisa quantitativa, de caráter bibliográfico e de campo, este estudo buscou avaliar o impacto do Materialismo na Simplicidade Voluntária - um dos estilos de vida de Anticonsumo - e o papel moderador do Autocontrole e da Orientação a Longo Prazo em estudantes universitários do curso de Administração. Os resultados da pesquisa apontaram correspondência negativa entre Simplicidade Voluntária e Materialismo, negando a estima exagerada a bens e posses e a supervalorização do consumo. Sugeriu-se, assim, que Simplicidade Voluntária não possui relação significante com o Autocontrole ou com a Orientação a Longo Prazo. A partir dos achados, é possível definir um início para a expansão do entendimento sobre o indivíduo detentor de um (ou mais) novo perfil de consumo, que aborda o mundo e as relações que o permeiam diferentemente do perfil consumista considerado habitual. Espera-se que este trabalho estimule estudos futuros, permitindo análises mais complexas sobre o comportamento de Anticonsumo e ampliando o debate sobre o tema no Marketing.

Palavras-chave: Anticonsumo, Materialismo, Simplicidade Voluntária, Sustentabilidade, Comportamento do Consumidor.

1 Possui graduação em Publicidade e Propaganda pela Universidade de Fortaleza - UNIFOR, Graduação em Administração e Mestrado em Administração e Controladoria pela Universidade Federal do Ceará - UFC. Atualmente é doutoranda em Administração pela Universidade de São Paulo - USP. São Paulo, São Paulo - Brasil. E-mail: sofiasbf@gmail.com

2 Possui graduação em Matemática Aplicada e Comp. - Prob. e Estatística pelo Instituto Superior Técnico da UTL, IST, Portugal, aperfeiçoamento em métodos estatísticos avançados em Biologia e Saúde pela Faculdade de Ciências da Universidade de Lisboa, mestrado em Gestão Empresarial pela Faculdade de Economia da Universidade do Algarve e doutorado em Estatística e Inv.Operacional -Prob. e Estatística pela faculdade de Ciências da Universidade de Lisboa. Fortaleza, Ceará - Brasil. E-mail: mdpedro@gmail.com

3 Possui graduação em Administração de Empresas e mestrado em Administração e Controladoria pela Universidade Federal do Ceará - UFC. Fortaleza, Ceará - Brasil. Email: gisellecqueiroz@gmail.com

4 Possui graduação em Publicidade e Propaganda pela Universidade de Fortaleza -UNIFOR, mestrado em Administração e Controladoria pela Universidade Federal do Ceará. Atualmente é professor titular da Faculdade 7 de Setembro e sócio-fundador da empresa Logovia. Fortaleza, Ceará - Brasil. E-mail: davidecastrorocha@gmail.com 


\section{ABSTRACT}

The role of consumption culture has been increasingly questioned as a societies' vector of happiness and well-being. The idea of renouncing the market codes imposed to the community gains emphasis in favor of the adoption of values of internal and external harmony with the environment. Thus, through a quantitative study, of bibliographical and field research order, this study sought to evaluate the impact of Materialism in Voluntary Simplicity - one of the lifestyles of Anti-consumerism - and the moderating role of Self-control and Long-Term Orientation in Bsc. students. The survey results showed a negative correlation between Voluntary Simplicity and Materialism, denying exaggerated desire of goods and possessions and overvaluation of consumption. It was suggested that Voluntary Simplicity does not have a significant relationship with Self-control or the Long Term Orientation. From the findings, it is possible to define a initial expansion of the understanding of the individual holder of one (or more) new consumption behavior profile that sees differently the world and the relationships that permeates it. It is expected that the work will stimulate future studies, allowing more complex analyzes on the behavior of Anti-consumerism and will broad the debate on the topic among Marketing scholars.

Keywords: Anti-consumption. Materialism. Voluntary Simplicity. Sustainability. Consumer Behavior.

\section{INTRODUÇÃO}

A cultura de consumo, em âmbito global, ao invés de corresponder às demandas de preenchimento, felicidade e satisfação, tem comprometido diversos fatores vinculados ao bem-estar da sociedade (ALEXANDER, 2011). A partir desse panorama, questiona-se o papel regido pelo consumo nas vidas dos indivíduos e se, de fato, ele é a solução para a demanda da sociedade ou, ainda, para as suas necessidades.

Para entender o consumo e suas consequências, seja em contexto pessoal, social, acadêmico ou mercadológico, deve-se buscar um olhar holístico sobre o fenômeno. Além de conhecer com profundidade o comportamento de consumo, é necessário entender o não consumo para compreender as faces positivas e negativas da questão (GOULD; HOUSTON; MUNDT, 1997) e, assim, não limitar a compreensão acerca dos consumidores.

Nesse contexto, Marion (2003) discorre sobre o crescente número de indivíduos críticos que refutam os códigos mercadológicos impostos à comunidade e para aqueles que são compelidos a abraçar valores que não estão em harmonia com o que pensam e sentem e com o ambiente que os cerca. A partir desses fatos, delineia-se a necessidade de entender melhor as variáveis envolvidas nessa atividade de não consumo, principalmente no que concerne à Simplicidade Voluntária, estilo de vida genuinamente sustentável ou, em outras palavras, "modo de vida que é materialmente mais frugal, orientado para a ecologia, direcionado para o íntimo" (ELGIN, MITCHELL; 1977, p. 51), temática pouco abordada no contexto brasileiro. Ademais, os questionamentos são os mais diversos e merecem atenção por indicarem o caminho para uma seara em desenvolvimento e de grande potencial a ser explorada.

Tendo em vista a importância dessa temática, o presente estudo objetiva analisar a influência do Materialismo nos estilos de vida de Anticonsumo, em especial o estilo da Simplicidade Voluntária (SV) e o papel moderador do Autocontrole e da Orientação a Longo Prazo em estudantes de graduação da área da Administração. Busca-se, ainda, avaliar o Materialismo e a Simplicidade Voluntária (SV) em estudantes de Administração; analisar a relação entre a SV e o Autocontrole e Orientação a Longo Prazo; e avaliar a influência das variáveis moderadoras (Autocontrole e Orientação a Longo Prazo) na relação entre o Materialismo e o estilo de vida da Simplicidade Voluntária.

A relevância deste estudo é refletida em diferentes âmbitos: social, mercadológico e acadêmico. Concernente à esfera social, percebe-se o anticonsumo como uma importante fer- 
ramenta para a promoção da sustentabilidade e do consumo consciente. Em âmbito mercadológico, o estudo do anticonsumo permite o maior conhecimento sobre nichos de mercado e de soluções que correspondam à expectativa e à consciência do consumidor (e, também, do não consumidor). Sob a perspectiva acadêmica, espera-se que este estudo ajude a validar o modelo de antecedentes de estilos de vida e permita a idealização e realização de novas pesquisas a partir dos resultados obtidos.

\section{REVISÃO BIBLIOGRÁFICA}

\subsection{Materialismo}

De acordo com Belk (1991, p. 141), "quanto mais acreditamos que possuímos ou somos possuídos por um objeto, mais parte do nosso eu ele se torna". Além disso, pesquisas demonstram que os consumidores, de modo geral, estão cada vez mais materialistas (BELK, 1991; WATSON, 2003; RICHINS, 2004; KILBOURNE; GRUNHAGEN; FOLEY, 2005; GROHMANN et al., 2012).

Paralelamente a essa perspectiva crítica da contemporaneidade, introduz-se a importância do estudo do construto Materialismo. Pesquisado em diversos contextos, sejam eles nacionais e/ou internacionais, trata-se de um conceito que permite inúmeras conjunturas. Sabe-se ainda que, pelo seu estudo em diferentes épocas, os autores não determinaram uma definição uníssona para o termo (KILBOURNE; GRUNHAGEN; FOLEY, 2005).

Dentre as definições mais propagadas e utilizadas no âmbito do comportamento do consumidor, tem-se a de Belk $(1984,1985)$, que afirma que materialismo, tratando-se de um traço de personalidade, é a importância que determinado consumidor atribui às posses materiais: nos níveis mais altos, assumem posição central no cotidiano e são constantemente consideradas como fontes de satisfação ou até de insatisfação para o indivíduo.

Além da abordagem de Belk (1984, 1985), Richins e Dawson (1992, p. 304) revelam, em seu estudo com 690 norte-americanos, a segmentação do construto em três dimensões: centralidade, felicidade e sucesso - sendo esta última determinada pela quantidade e qualidade das posses obtidas. A escala desenvolvida pelos autores, baseada nos conceitos de posse e aquisição, foi reduzida na proposta de Richins (2004) de 18 para 15 itens e, também, para nove itens e seis itens com o intuito de mensurar o materialismo como indicador global.

A relevância das posses na vida dos indivíduos, conjugada com a percepção dos malefícios do consumo exacerbado, resultou em estudos que relacionam o materialismo ao anticonsumo e aos seus diferentes estilos de vida (RICK; CRYDER; LOEWENSTEIN, 2007; ETZIONI, 1998; ALEXANDER, 2011; NEPOMUCENO, 2012). Este estudo, em particular, aborda o estilo de vida da Simplicidade Voluntária por ser um estilo que, de acordo com a literatura estudada, apresenta maior relação com um estilo de vida adverso à retenção de posses e mais voltado a questões relativas ao meio ambiente e ao papel do indivíduo nele inserido (KILBOURNE, 1992; CRAIG-LEES; HILL, 2002; ZAVESTOKI, 2002).

Em síntese, as teorias mencionadas encadeiam a hipótese que segue:

H1: O Materialismo está negativamente relacionado à Simplicidade Voluntária.

Para aprofundar o conhecimento nesse ponto, explora-se, a seguir, a questão do anticonsumo, sua origem e suas definições, bem como os estudos já realizados, com enfoque no estilo da Simplicidade Voluntária. 


\subsection{Anticonsumo}

De modo a refutar a lógica mercadológica muitas vezes impositiva, uma quantidade cada vez maior de pessoas tem percebido que riqueza e posses materiais não compensam os sentimentos de estresse, tristeza e infelicidade (ZAVESTOSKI, 2002). Paralelo a isso, percebe-se, sobremaneira, que uma parcela dos indivíduos se encontra cada vez mais crítica, motivada e compelida a manifestar-se em prol de seu livre arbítrio, da autonomia de suas decisões e do controle de seu futuro (MARION, 2003).

Sabe-se que a maioria das pesquisas permanece com foco no consumo e nos seus aspectos positivos e que o interesse em temas relacionados com o anticonsumo ou a não escolha ainda é perifericamente explorado, apesar do aumento do interesse sobre o tema nas últimas décadas (SUAREZ; CHAUVEL; CASOTTI, 2012). Até meados da década de 1990, ainda se tratava de um tópico negligenciado e pouco explorado (BANISTER; HOGG, 2004).

Autores como Iver e Muncy (2009) e Kozinets, Handelman e Lee (2010) mencionam um perfil de pessoas "simplificadoras" que baseiam seus estilos de vida na socialização de valores antimaterialistas. É interessante notar, dessa maneira, a existência de diferentes comportamentos de anticonsumo.

De acordo com Matos (2011, p. 2), o anticonsumo engloba desde a "gama heterogênea de atividades políticas, sociais e de consumo envolvendo movimentos informais e individualistas como os simplificadores voluntários até mesmo movimentos organizados de larga escala, como os protestos antiglobalização". A pesquisa concernente ao anticonsumo abrange o estudo das razões pelas quais o consumidor falhou em comprar ou consumir e dos motivos pelos quais ele optou pelo não consumo (GOULD; HOUSTON; MUNDT, 1997).

Dentre as mais variadas abordagens acerca do anticonsumo, pode-se destacar aquelas que dizem respeito à resistência do consumidor (FOURNIER, 1998; MATOS, 2011; SILVA; COSTA; CARVALHO, 2011), aos movimentos contraculturais (ZAVESTOSKI, 2002; DINIZ, 2008), ao consumo ético (SHAW; NEWHOLM, 2002), ao não consumo (HOGG, 1998; STAMMERJOHAN; WEBSTER, 2002) e aos movimentos antimarcas (HOLLENBECK; ZINKHAN, 2010; ALBUQUERQUE et al., 2010).

Nesse contexto, destaca-se, em âmbito internacional, a pesquisa de Gould et al. (1997), que apresenta uma diferenciação entre o não consumo passivo e o ativo (antiescolha), sendo este caracterizado por um indivíduo que apenas não tenta consumir (ignora ou rejeita propostas de consumo) e aquele por um que "tenta não tentar" (realiza esforços para não consumir).

Em um segundo momento, Iver e Muncy (2009) apresentam uma reflexão quanto à motivação para o anticonsumo e categorizam quatro diferentes tipos de anticonsumidores: 1) aqueles preocupados com o impacto global do consumo; 2) aqueles que optam pela Simplicidade Voluntária, ou seja, pelo anticonsumo; 3) aqueles proativos e/ou ativistas de mercados; e 4) os consumidores antifiéis. Cherrier (2009), por sua vez, analisa dois movimentos relevantes do campo do anticonsumo (Simplicidade Voluntária e culture jamming), articulando-os às duas tipologias de identidade (de herói e de projeto, respectivamente).

No contexto nacional, tem-se o recente estudo de Suarez, Chauvel e Casotti (2012), que traz à tona três tipos de abandono (contingencial, posicional e ideológico) e demonstra que não se trata apenas de "um distanciamento de significados negativos, com o objetivo de proteger a autoestima" (p. 411). Pode-se citar ainda Matos (2011), que estudou os antecedentes do conceito de resistência no campo do anticonsumo.

Ao enfocar as pesquisas sobre anticonsumo e estilo de vida da Simplicidade Voluntária, como já foi mencionado, torna-se necessário, em um primeiro momento, entender a razão pela 
qual isso é relevante e o que é estilo de vida.

Grisi (1986) aponta que o estilo de vida pode ser abordado enquanto variável dependente de outras, como personalidade, status social e fatores demográficos, por exemplo. Entretanto, a autora discute que, para a função de segmentação de mercado, o estilo de vida possui grande utilidade como variável independente, pois auxilia a explicar o comportamento do consumidor. É, assim, a partir dos valores, das atitudes e dos comportamentos diários que os estilos de vida são evidenciados e compartilhados.

Apesar de viver em um mundo materialista, existe uma parcela de consumidores que adotam estilos de vida pautados na redução do consumo ou no anticonsumismo (NEPOMUCENO, 2012). A partir da compreensão acerca de estilos de vida, é necessário, todavia, entender o estilo elencado para este estudo (SV), com o intuito de amparar o desenvolvimento da pesquisa e contextualizá-lo no modelo proposto.

O conceito de Simplicidade Voluntária não é recente e foi definido, primeiramente, como uma pureza, honestidade e sinceridade de propósito que buscasse o distanciamento de comportamentos pautados em posses materiais (GREGG, 1936). Elgin e Mitchell (1977) relacionam esse estilo de vida ao senso de urgência e responsabilidade social que, há algumas décadas, era escasso. O senso de urgência, conforme informam os autores, deriva dos diversos problemas da sociedade, como as previsões de uma futura pane de energia, a depreciação do meio ambiente, entre outros.

A SV, enquanto estilo de vida, é colocada em prática com a minimização do consumo de bens materiais, de modo a exercitar a autossuficiência e desenvolver o intelecto e outras facetas não materiais inerentes à existência humana. Também conhecida como downshifting (redução de marchas, de velocidade ou intensidade, em inglês) ou "vida simples", é um estilo de vida que se opõe aos elevados padrões das sociedades de consumo e implica uma vida mais simples com o consumo apenas do que é necessário (ZAVESTOSKI, 2002). Gregg (1936), Elgin e Mitchell (1977) e Shama (1981) ressaltam que a caracterização do movimento da SV baseia-se em seis valores principais: 1) Simplicidade Material; 2) Escala Humanizada, ou seja, humanização da rotina, inclusive do ambiente de convivência e da natureza (meio ambiente); 3) Tecnologia Apropriada ao invés da aplicação automática da mais alta tecnologia; 4) Autodeterminação; 5) Preocupação Ambiental; e 6) Crescimento Pessoal, isto é, o desejo de viver uma vida mais voltada ao desenvolvimento interno.

Como muitos autores lembram ao considerarem o estilo de vida da SV (KILBOURNE, 1992; CRAIG-LEES; HILL, 2002; ZAVESTOKI, 2002), não se trata de viver com base na pobreza, mas de estimular uma redefinição de prioridades com base na busca do verdadeiro eu ou, de forma mais objetiva, da "simplicidade da individualidade". Diante disso, a revisão teórica desta seção sugere as seguintes hipóteses:

H2: A Simplicidade Voluntária não possui relação significativa com a Orientação a Longo Prazo (LTO) nem com o Autocontrole.

H3: O Materialismo está negativamente relacionado à Simplicidade Voluntária, independentemente da influência das variáveis moderadoras Autocontrole e LTO.

\subsection{Autocontrole}

Rothbaum, Weisz e Snyder (1982) definem autocontrole como a capacidade de mudar e adaptar-se, com o objetivo de estar mais bem ajustado ao mundo. Uma das características centrais do autocontrole é a habilidade de direcionar ou mudar o seu próprio comportamento, interrompendo aqueles que são indesejados.

De acordo com a definição de Stammerjohan e Webster (2002), o autocontrole pode ser 
considerado uma variável individual que molda as ações e os comportamentos. Sabe-se, ainda, que pode se referir à habilidade de inibir, desvencilhar ou alterar respostas indesejadas para si ou para os outros, seja quanto a um hábito, um processo ou uma situação (SCHMEICHEL; BAUMEISTER, 2004). Nesse sentido, Hoch e Loewenstein (1991) encontraram uma relação positiva entre a dimensão individual do autocontrole e o comportamento de anticonsumo. Ressalta-se, entretanto, a afirmação de Hoch e Lowenstein (1991), conforme expõe Nepomuceno (2012), de que o indivíduo pode ter uma alta pontuação em autocontrole não exercida, necessariamente, no campo do consumo, mas em outras esferas da sua vida.

Já os estudos de Poynor e Haws (2009), por exemplo, indicam que os indivíduos com menos pontuação de autocontrole classificam mais itens como uma necessidade, sendo mais materialistas ou ligados a posses e bens. Rose (2007), por sua vez, correlaciona positivamente o materialismo e a compulsão de compra.

\subsection{Orientação a Longo Prazo}

De acordo com alguns autores, LTO (long-term orientation ou orientação a longo prazo) pode ser definida como um valor cultural no qual o tempo é visto de forma holística, em que se valoriza tanto o passado quanto o futuro em vez de julgar a importância de ações apenas por seus efeitos imediatos ou a curto prazo.

Os trabalhos de Hoch e Lowenstein (1991), Howlett, Kees e Kemp (2008), Mogilner e Aaker (2009) e Nepomuceno (2012) pesquisaram diferentes facetas da influência da orientação do tempo sobre o comportamento do consumidor quanto à preferência e compra de produtos. Apesar disso, sabe-se que a relação entre orientação a longo prazo e anticonsumo ainda é pouco estudada na literatura.

Acerca da Simplicidade Voluntária, Nepomuceno (2012) expõe que, por se tratar de um estilo que é, por definição, anticonsumista, pensa-se que não haverá correlação significativa com LTO, pois não importará a orientação a curto ou a longo prazo. Percebe-se, portanto, ao observar a influência das duas variáveis moderadoras, que elas possuem papéis semelhantes quando relacionadas ao construto Simplicidade Voluntária. Diante disso, expõe-se, no Quadro 1, o resumo das hipóteses que orientaram este estudo.

Quadro 1 - Resumo das hipóteses do estudo

\begin{tabular}{|c|l|}
\hline Hipótese & \multicolumn{1}{c|}{ Resumo } \\
\hline H1 & O Materialismo está negativamente relacionado à Simplicidade Voluntária. \\
\hline H2 & $\begin{array}{l}\text { A Simplicidade Voluntária não possui relação significativa com a Orientação a Longo Prazo } \\
\text { (LTO) nem com o Autocontrole. }\end{array}$ \\
\hline H3 & $\begin{array}{l}\text { O materialismo está negativamente relacionado à Simplicidade Voluntária, independente- } \\
\text { mente da influência das variáveis moderadoras Autocontrole e LTO. }\end{array}$ \\
\hline
\end{tabular}

Fonte: dados da pesquisa.

O modelo utilizado na presente pesquisa baseou-se no trabalho de Nepomuceno (2012) sobre anticonsumo e estilos de vida aplicado em países como Canadá, Estados Unidos, Índia e Brasil, uma vez que o modelo vai além da relação direta entre materialismo e estilos de vida anticonsumistas (Figura 1). 
Figura 1 - Modelo utilizado no estudo

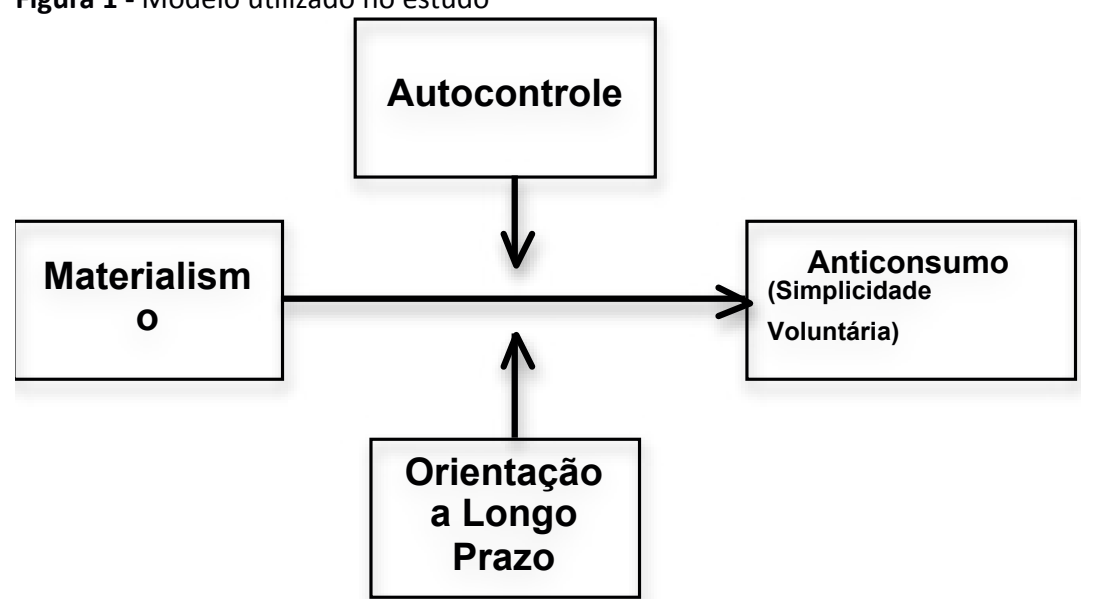

Fonte: adaptado de Nepomuceno (2012).

Dessa forma, no modelo apresentado na Figura 1, percebe-se o materialismo como variável independente, enquanto que o estilo de vida de anticonsumo escolhido (SV) é considerado variável dependente.

\section{METODOLOGIA}

Este estudo pode ser definido como de natureza essencialmente quantitativa (COLLIS; HUSSEY, 2005), caracterizando-se como descritivo, no que diz respeito aos objetivos (MALHOTRA,2011) e bibliográfico (dados secundários) e de campo (dados primários, advindos de livros, jornais, periódicos e anais de ventos), no que se refere aos meios (MALHOTRA, 2011).

O universo da pesquisa de campo ou população-alvo foi composto de alunos universitários da área da gestão. Outros estudos acerca de anticonsumo ou consumo e materialismo também se utilizaram de amostras de estudantes, como o de Zavestoski (2002) e o de Nepomuceno (2012).

No caso deste estudo, foi utilizada uma amostragem não probabilística, por julgamento, baseada no conhecimento do próprio pesquisador (HAIR JR et al., 2010). As unidades amostrais escoIhidas para representar o estudo foram alunos do curso de Administração da Universidade Federal do Ceará (UFC), pertencentes à Faculdade de Economia, Administração, Atuária e Contabilidade (FEAAC).

Em relação ao método de coleta ou levantamento de dados, foi aplicado, em julho de 2013, um questionário padronizado a alunos da área de Administração, método comumente utilizado em pesquisas descritivas e que costuma garantir "a comparabilidade dos dados, aumentar a velocidade e a precisão do registro e facilitar o processamento de dados" (MALHOTRA, 2011, p. 290), conforme o resumo apresentado no Quadro 2.

Quadro 2 - Resumo dos aspectos referentes à coleta de dados

\begin{tabular}{|l|l|}
\hline Número de questionários coletados & 217 questionários \\
\hline Locais de coleta & FEAAC - UFC \\
\hline Forma de coleta & Questionário autoaplicado \\
\hline Tempo de preenchimento do questionário & $10-15$ minutos \\
\hline Período de coleta & Julho de 2013 \\
\hline Monitoramento de coleta & Dois pesquisadores e um auxiliar \\
\hline Seleção da amostra & Por julgamento (proposital) \\
\hline
\end{tabular}

Fonte: informações sobre a pesquisa. 
O questionário foi formado por afirmações estruturadas, com um conjunto preestabelecido de formato de respostas para cada construto. Com base no trabalho realizado por Nepomuceno (2012), o materialismo foi mensurado com a escala de Richins (2004), a partir da adaptação de Garcia (2009); para a Simplicidade Voluntária, foi usada a escala de Iwata (1999, 2006); para o autocontrole, foi utilizado o estudo de Tangney, Baumeister e Boone (2004); e, para a orientação a longo prazo, optou-se por Bearden, Money e Nevins (2006).

Para a análise dos dados coletados, foram realizados procedimentos estatísticos descritivos e inferenciais e análise multivariada dos dados. Esta última foi feita por meio do software estatístico Statistical Package for the Social Sciences (SPSS) 20.0.

Para o tratamento dos dados, foi utilizada uma sequencia lógica de análise. Primeiramente, realizou-se a análise descritiva das variáveis sociodemográficas dos estudantes. Em seguida, foi realizada a análise descritiva das variáveis de medida dos vários construtos em estudo e, após, foi realizada a análise fatorial para criar novas categorias ou fatores com base nas novas variáveis representativas dos construtos do modelo. A análise fatorial foi aplicada à matriz de correlações, com extração dos fatores pelo método das componentes principais, seguida de uma rotação Varimax. Desta análise resultou uma variável para representar cada fator, determinada pela média ponderada dos itens correspondentes. Posteriormente, procedeu-se à análise descritiva dessas variáveis, bem como à análise de correlação entre as variáveis, o que permitiu validar ou não as hipóteses H1 e H2. Por fim, utilizou-se do método de regressão linear para compreender a avaliação e mensuração de variáveis explicativas em relação a apenas uma dependente métrica que configurará o fenômeno do anticonsumo, sobre o qual trata esta pesquisa. A inclusão de termos de interação das variáveis referentes ao materialismo, como o autocontrole e a orientação a longo prazo, permitiu avaliar a significância dessas variáveis como moderadoras da influência do materialismo no anticonsumo e, consequentemente, a hipótese H3.

\section{ANÁLISE E DISCUSSÃO DOS RESULTADOS}

\subsection{Caracterização da amostra: perfil sociodemográfico}

A análise descritiva torna-se necessária por resumir uma quantidade significativa de informação em um menor número de valores e medidas. Buscou-se, assim, exibir os dados sociodemográficos referentes às amostras e revelar, de forma sucinta, o perfil dos respondentes. Dos 213 respondentes válidos, 98,2\% são brasileiros natos, 57,9\% são homens, 61,3\% têm entre 21 e 25 anos, 90,8\% são solteiros, 70,5\% trabalham meio período ou estagiam (70,5\%), 29,6\% ganham entre um e dois salários mínimos e $23,7 \%$ têm uma renda familiar que varia entre seis e 10 salários mínimos.

\subsection{Análise descritiva dos construtos}

Percebe-se, a partir da análise dos valores do construto Materialismo, que a maioria das médias das variáveis encontra-se centralizada em torno do valor "3". Deve-se ressaltar, entretanto, que a média mais baixa corresponde a M6 ("Quando estou triste, faço compras para me sentir melhor") referente à dimensão felicidade, e que a mais alta foi M12 ("Eu sinto prazer em fazer compras") relativa à centralidade das posses materiais no estilo de viver a vida por parte do indivíduo. De forma descendente, M2 e M4 apresentaram pontuações parecidas (3,31 e 3,33, respectivamente) e ambas estavam relacionadas à ideia de uma prospecção de vida melhor do 
que a atual por meio da obtenção de produtos que não possuem e/ou que não ainda podem obter. Todas as variáveis apresentam desvios padrão (DP) próximo de 1, não tendo sido registradas diferenças consideráveis nas suas dispersões.

A Orientação a Longo Prazo (LTO) apresentou médias muito favoráveis ao planejamento - orientação com base no futuro e longo prazo. Percebeu-se, também, uma uniformidade nas médias e nos desvios-padrão desse construto.

Realizou-se, ainda, a análise descritiva do construto Autocontrole. Dessa forma, ao observar as médias e os desvios-padrão das 13 variáveis elencadas, nota-se uma preponderância ao autocontrole, visto que todas as médias pontuaram acima de 4, com exceção de SC5 ("Eu faço certas coisas que me prejudicam, se elas forem divertidas"), que apresentou um valor de 2,83.

Por fim, tem-se a análise do construto Simplicidade Voluntária (SV), o qual apresentou médias relativamente abaixo da medida central. Das variáveis que apresentaram médias mais baixas, podem-se destacar aquelas relativas à autossuficiência, SV6 e SV7, que afirmam, respectivamente: "Gostaria de plantar minha própria comida no futuro" e "No futuro, eu produzirei meus próprios bens (tais como roupas e ferramentas).

Em suma, acredita-se que, apesar da heterogeneidade do construto, a média da maioria das variáveis tende a ser avessa à Simplicidade Voluntária, que, conforme ratificado no referencial teórico, trata-se do estilo de vida genuinamente anticonsumista.

\subsection{Análise Fatorial dos construtos}

Foram analisados os construtos da presente pesquisa. A adequação desta análise ao conjunto de dados foi avaliada com base em índices como o Kaiser-Meyer-Olkin (KMO), para mensurar a adequação da amostra, e o teste de esfericidade de Bartlett a partir do qui-quadrado observado, dos graus de liberdade e do valor $p<0,001$ (que foi comparado com um nível de significância de 0,05). Para a rotação dos fatores, foi utilizado o método Varimax (REIS, 2001; FÁVERO et al., 2009).

\subsubsection{Análise Fatorial: Materialismo}

A aplicação da AF às variáveis referentes ao materialismo revelou-se adequada, tendose obtido um KMO de 0,742, considerado médio por Fávero et al. (2004), e um valor de p inferior a 0,001 para o teste de esfericidade de Bartlett, conforme apresentado na Tabela 1.

Tabela 1 - KMO e teste da esfericidade de Bartlett do Materialismo

\begin{tabular}{cc|c}
\hline Kaiser-Meyer-Olkin & Medida de adequação da amostra &, 742 \\
\hline Teste de esfericidade de Bartlett & Qui-quadrado observado & 597,071 \\
\cline { 2 - 3 } & Graus de liberdade & 78 \\
\cline { 2 - 3 } & Valor $p$ &, 000 \\
\hline
\end{tabular}

Fonte: dados da pesquisa.

O critério do autovalor superior a 1 (definido como Critério de Kaiser) para a escolha do número de fatores indicou a retenção de quatro fatores, que explicariam 59,833\% da variância dos dados. Contudo, o autovalor do quarto fator é de 1,008, ultrapassando ligeiramente o valor 1 estabelecido como critério (WORTHINGTON; WHITTAKER, 2006; MALHOTRA, 2011).

A revisão bibliográfica sobre materialismo ou, mais especificamente, a escala sobre materia- 
lismo de Richins (2004), adaptada por Garcia (2009), aponta para a existência de três fatores, motivo pelo qual se optou por este número de fatores, decrescendo a variância explicada para 52,083\%. É importante referir ainda que as variáveis 11, 16 e 17 são invertidas, da mesma forma que as variáveis 6 e 12 foram retiradas uma a uma por apresentarem comunalidades inferiores a 0,4 (proporção de variabilidade total de cada variável explicada por todos os fatores retidos), critério adotado nesta pesquisa e também estabelecido pela literatura (WORTHINGTON; WHITTAKER, 2006).

As cargas fatoriais estimadas a partir da rotação dos fatores são apresentadas na Tabela 2.

Tabela 2 - Cargas Fatoriais do Materialismo após rotação Varimax

\begin{tabular}{|c|c|c|c|c|}
\hline \multirow{2}{*}{ Variável original } & \multicolumn{3}{|c|}{ Fator } & \multirow{2}{*}{ Comunalidade } \\
\hline & Centralidade & Felicidade & Sucesso & \\
\hline M7i & ,726 & ,164 &,- 169 & 0,582 \\
\hline M1i & 699 &,- 001 &,- 182 & 0,522 \\
\hline M13i & 697 &, 250 &,- 010 & 0,549 \\
\hline M12 & ,669 &, 006 & 179 & 0,479 \\
\hline M15 & ,604 & ,133 & ,279 & 0,460 \\
\hline M6 &, 544 &,- 091 & ,319 & 0,406 \\
\hline M10 & ,011 & ,776 & ,102 & 0,612 \\
\hline M4 & ,117 & ,717 & ,300 & 0,618 \\
\hline M9i &, 031 & ,686 &,- 141 & 0,492 \\
\hline M2 & 197 &, 656 & ,151 & 0,492 \\
\hline M5 & ,169 & ,018 & ,737 & 0,572 \\
\hline M8 &, 040 &, 053 & ,704 & 0,499 \\
\hline M3 &,- 112 &, 250 & ,642 & 0,487 \\
\hline \%variância explicada & 25,229 & 15,188 & 11,665 & \\
\hline \%variância explicada acumulada & 25,229 & 40,417 & 52,083 & \\
\hline
\end{tabular}

Fonte: dados da pesquisa.

É válido salientar que todas as variáveis, por apresentarem carga fatorial acima de 0,50, são consideradas estatisticamente significantes por Hair et al. (2010). Como o tamanho da amostra do estudo ultrapassou a quantidade de 200 unidades amostrais, os autores afirmam que o mínimo pode ser até de 0,40.

\subsubsection{Análise Fatorial: Orientação a Longo Prazo (LTO)}

Trata-se de um construto unidimensional. Assim como ocorreu com o construto Materialismo, o LTO apresentou KMO 0,745 e o valor de $p<0,001$, conforme explicitados na Tabela 3.

Tabela 3 - KMO e teste da esfericidade de Bartlett do LTO

\begin{tabular}{cc|c}
\hline Kaiser-Meyer-Olkin & Medida de adequação da amostra & ,745 \\
\hline Teste de esfericidade de Bartlett & Qui-Quadrado observado & 188,233 \\
\cline { 2 - 3 } & Graus de liberdade & 6 \\
\cline { 2 - 3 } & Valor $p$ &, 000 \\
\hline
\end{tabular}

Fonte: dados da pesquisa.

Apresentou, ainda, em consonância com a Tabela 4, todas as comunalidades acima de 0,4 (o menor valor foi de 0,524), além da variância explicada de 56,93\%. 
SOFIA BATISTA FERRAZ

SILVIA MARIA DIAS PEDRO REBOUÇAS GiSElle CAVALCANTE QUEIROZ

DAVI DE CASTRO ROCHA

Tabela 4 - Cargas Fatoriais do LTO após rotação Varimax

\begin{tabular}{c|c|c}
\hline Variável original & Fator & Comunalidade \\
\cline { 2 - 3 } & Planejamento & 0,598 \\
\hline LTO3 &, 773 & 0,596 \\
\hline LTO2 &, 772 & 0,560 \\
\hline LTO1 &, 748 & 0,524 \\
\hline \% variância explicada &, 724 & \\
\hline
\end{tabular}

Fonte: dados da pesquisa.

O fator planejamento unidimensional, no caso da presente pesquisa, advém do trabalho de Bearden, Money e Nevins (2006) com adaptação de Nepomuceno (2012). Não houve escalas invertidas e todas as cargas fatoriais obtiveram peso acima de 0,724 , o que indica que se trata de um fator estatisticamente significante.

\subsubsection{Análise Fatorial: Autocontrole (SF)}

O Autocontrole, de acordo com a Tabela 5, apresentou um valor do KMO de 0,723 e um valor de $p$ inferior a 0,001, o que torna a aplicação da análise fatorial adequada.

Tabela 5 - KMO e teste da esfericidade de Bartlett do SF

\begin{tabular}{cc|c}
\hline Kaiser-Meyer-Olkin & Medida de adequação da amostra &, 723 \\
\hline Teste de esfericidade de Bartlett & Qui-quadrado observado & 467,548 \\
\cline { 2 - 3 } & Graus de liberdade & 66 \\
\cline { 2 - 3 } & Valor $p$ &, 000 \\
\hline
\end{tabular}

Fonte: dados da pesquisa.

A literatura concernente ao autocontrole, principalmente o trabalho de Tangney, Baumeister e Boone (2004), ao elaborar a "Brief Self Control Scale", demonstrou a existência de quatro fatores, todos com autovalor superior a 1, como pode ser verificado na Tabela 6 . A variância explicada acumulada do construto foi de $58,82 \%$, e todas as cargas fatoriais apresentaram valor acima de 0,50 (a mais baixa foi a SC6, com 0,503). Vale ressaltar, ainda, que a variável 2 foi eliminada por apresentar comunalidade inferior a 0,4 , não sendo, portanto, aprovada no requisito estabelecido.

Tabela 6 - Cargas Fatoriais do SC após rotação Varimax

\begin{tabular}{|c|c|c|c|c|c|}
\hline \multirow[b]{2}{*}{ Variável original } & \multicolumn{4}{|c|}{ Fator } & \multirow[b]{2}{*}{$\begin{array}{c}\text { Comunali- } \\
\text { dade }\end{array}$} \\
\hline & $\begin{array}{c}\text { Ações } \\
\text { deliberadas }\end{array}$ & $\begin{array}{l}\text { Autodisci- } \\
\text { plina }\end{array}$ & $\begin{array}{l}\text { Hábitos } \\
\text { saudáveis }\end{array}$ & Confiabilidade & \\
\hline SC5i &, 800 &, 098 &, 204 &,- 081 & 0,698 \\
\hline SC12i & 786 & 013 & -108 & 246 & 0.690 \\
\hline SC4i & .602 & 287 & 312 &,- 044 & 0,545 \\
\hline SC3i & 111 & .702 & 309 & -.024 & 0,601 \\
\hline SC7i &,- 019 & 648 &,- 110 & 385 & 0,580 \\
\hline SC10i & 149 & 646 & .059 & 008 & 0,443 \\
\hline SC9i & 386 & 527 & 249 & 116 & 0,503 \\
\hline SC11 & 027 & 082 & .747 & 078 & 0,571 \\
\hline SC8 & 138 & 220 & 726 & -.035 & 0,596 \\
\hline SC6 & 155 & -.464 & 503 & 239 & 0.549 \\
\hline SC1 &,- 043 & .069 & .163 & 811 & 0,692 \\
\hline SC13i & .473 & 084 &,- 065 & 597 & 0,592 \\
\hline \%variância explicada & 26,412 & 12,051 & 11,164 & 9,200 & \\
\hline $\begin{array}{c}\text { \%variância explicada } \\
\text { acumulada }\end{array}$ & 26,412 & 38,463 & 49,627 & 58,827 & \\
\hline
\end{tabular}

Fonte: dados da pesquisa. 
Estabeleceu-se, assim, a existência de quatro fatores: o primeiro refere-se às Ações Deliberadas (SC4, SC5 e SC12, todos invertidos); o segundo diz respeito à Autodisciplina (SC3, SC7, SC9 e SC10, também invertidos); o terceiro respeito é relativo aos Hábitos Saudáveis (SC6, SC8 e SC11); e o quarto é referente à Confiabilidade e Autonomia (SC1 e SC13 invertida).

\subsubsection{Análise Fatorial: Simplicidade Voluntária}

A Simplicidade Voluntária obteve KMO de 0,715 e, assim como os outros construtos avaliados anteriormente, $p<000,1$. A Tabela 7 organiza esses dados da seguinte maneira:

Tabela 7 - KMO e teste da esfericidade de Bartlett da Simplicidade Voluntária

\begin{tabular}{cc|c}
\hline Kaiser-Meyer-Olkin & Medida de adequação da amostra & \multicolumn{1}{c}{, 715} \\
\hline Teste de esfericidade de Bartlett & Qui-quadrado observado & 435,670 \\
\cline { 2 - 3 } & Graus de liberdade & 36 \\
\cline { 2 - 3 } & Valor $p$ &, 000 \\
\hline
\end{tabular}

Fonte: dados da pesquisa.

A partir do critério do autovalor superior a 1, foi sugerida a existência de três fatores, coerente com a teoria sobre o tema proposta por Iwata (2006). Foi retirada do construto apenas a variável 4 , devido ao seu valor inferior a 0,4 , conforme a Tabela 8.

Tabela 8 - Cargas Fatoriais da Simplicidade Voluntária após rotação Varimax

\begin{tabular}{c|c|c|c|c}
\hline \multirow{2}{*}{$\begin{array}{c}\text { Variável } \\
\text { original }\end{array}$} & $\begin{array}{c}\text { Ponderação no ato } \\
\text { de comprar }\end{array}$ & $\begin{array}{c}\text { Desejo por vida } \\
\text { simples }\end{array}$ & Autossuficiência & Comun. \\
\cline { 2 - 5 } & $\mathbf{8 0 5}$ &, 112 &, 147 & 0,683 \\
\hline VS3 &, $\mathbf{8 0 2}$ &, 115 &,- 057 & 0,660 \\
\hline VS2 & $\mathbf{7 5 0}$ &, 019 &, 194 & 0,600 \\
\hline VS5 & $\mathbf{5 8 4}$ &, 484 &, 054 & 0,578 \\
\hline VS1 &,- 059 &, 694 &, 007 & 0,486 \\
\hline VS10i &, 183 &, 689 &, 038 & 0,509 \\
\hline VS9 &, 228 &, 626 &, $\mathbf{8 7 9}$ & 0,536 \\
\hline VS8 &, 091 &, 033 & $\mathbf{0 , 7 8 2}$ \\
\hline VS7 &, 099 &, 157 & $\mathbf{1 2 , 6 0 5}$ & $\mathbf{0 , 7 2 1}$ \\
\hline VS6 & $\mathbf{3 3 , 8 8 4}$ & $\mathbf{1 5 , 2 2 5}$ & $\mathbf{6 1 , 7 1 4}$ & \\
\hline $\begin{array}{c}\text { \%variância } \\
\text { explicada }\end{array}$ & $\mathbf{3 3 , 8 8 4}$ & $\mathbf{4 9 , 1 0 9}$ & & \\
\hline $\begin{array}{c}\text { \%variância } \\
\text { explicada } \\
\text { acumulada }\end{array}$ & & & & \\
\hline
\end{tabular}

Fonte: dados da pesquisa.

Com base no trabalho de Iwata (2006), divide-se o construto em três fatores: Ponderação no ato de comprar (SV1, SV2, SV3 e SV5); Desejo por uma vida simples (SV8, SV9 e SV10); e Autossuficiência (V6 e V7).

\subsection{Análise de Correlação}

Nesta subseção, são apresentadas as correlações com base nos construtos evidenciados ao longo do trabalho. Busca-se, assim, avaliar as hipóteses sugeridas que podem ser respondidas 
a partir das correlações significantes estabelecidas (a 1 ou 5\%).

É interessante atentar que, de acordo com Hair et al. (2010), o coeficiente de correlação de Pearson irá medir a associação linear existente entre duas variáveis (no caso, o coeficiente varia entre - 1 e 1, enquanto 0 significa a ausência de relação), o que significa que se trata de uma medida para avaliar a força de uma relação linear.

A primeira hipótese do estudo, referente às correlações existentes entre o materialismo e os estilos de anticonsumo, sugere relações negativas entre os construtos, conforme explicitado no referencial teórico desta pesquisa. $\mathbf{H 1}$ afirma existir correlação negativa entre Materialismo e Simplicidade Voluntária. Observa-se, na Tabela 9, que se trata de uma correlação negativa $(-0,401)$ e significante $(p<0,001)$. A hipótese, portanto, foi confirmada.

Tabela 9 - Análise de correlações com a Simplicidade Voluntária

\begin{tabular}{|c|c|c|c|c|c|}
\hline & & $\begin{array}{c}\text { Simplicidade } \\
\text { Voluntária }\end{array}$ & Ponderação & $\begin{array}{c}\text { Vida } \\
\text { Simples } \\
\end{array}$ & Autossuficiência \\
\hline \multirow{2}{*}{$\begin{array}{l}\text { Materialismo } \\
\text { (geral) }\end{array}$} & $\begin{array}{l}\text { Coef. Correlação de } \\
\text { Pearson }\end{array}$ &,$- 401^{* *}$ &,$- 385^{* *}$ &,$- 385^{* *}$ &,- 113 \\
\hline & Valor $p$ &, 000 &, 000 & ,000 & ,096 \\
\hline \multirow[t]{2}{*}{ Centralidade } & $\begin{array}{l}\text { Coef. Correlação de } \\
\text { Pearson }\end{array}$ &,$- 527^{* *}$ &,$- 670^{* *}$ &,$- 380^{* *}$ &,- 106 \\
\hline & Valor $\mathrm{p}$ &, 000 &, 000 &, 000 & ,118 \\
\hline \multirow{2}{*}{ Felicidade } & $\begin{array}{l}\text { Coef. Correlação de } \\
\text { Pearson }\end{array}$ &,$- 217^{* *}$ &,$- 185^{* *}$ &,$- 257^{* *}$ &,- 041 \\
\hline & Valor $\mathrm{p}$ & ,001 & ,006 &, 000 &, 546 \\
\hline \multirow[t]{2}{*}{ Sucesso } & $\begin{array}{l}\text { Coef. Correlação de } \\
\text { Pearson }\end{array}$ &,- 124 &, 004 &,$- 178^{* *}$ &,- 100 \\
\hline & Valor $p$ & ,067 & ,956 & ,008 & 141 \\
\hline
\end{tabular}

Fonte: dados da pesquisa.

Nota: ** Correlação significativa a um nível de significância de $1 \%$

* Correlação significativa a um nível de significância de 5\%

A H2, que diz respeito à ausência de relação significativa entre Simplicidade Voluntária e as variáveis moderadoras (Autocontrole e LTO), foi confirmada. Isso se deve ao fato de que, conforme a Tabela 10, o construto geral da SV não estabeleceu relação significante com nenhuma das variáveis de modo geral, apenas com a dimensão Confiabilidade do construto Autocontrole (,270).

Tabela 10 - Análise de correlações com a Simplicidade Voluntária e moderadoras

\begin{tabular}{|c|c|c|c|c|c|}
\hline & & $\begin{array}{c}\text { Simplicidade } \\
\text { Voluntária } \\
\text { (geral) }\end{array}$ & Ponderação & Vida Simples & Autossuficiência \\
\hline \multirow{2}{*}{ LTO } & $\begin{array}{l}\text { Coef. Correlação } \\
\text { de Pearson }\end{array}$ & , 074 &, $176^{* *}$ &,- 095 & ,070 \\
\hline & Valor $p$ & 279 & ,009 & ,162 & ,303 \\
\hline \multirow{2}{*}{$\begin{array}{l}\text { Autocontrole } \\
\text { (geral) }\end{array}$} & $\begin{array}{l}\text { Coef. Correlação } \\
\text { de Pearson }\end{array}$ & ,068 &, $225^{* *}$ &,- 081 &,- 002 \\
\hline & Valor $p$ & ,319 & ,001 & ,234 & ,976 \\
\hline \multirow{2}{*}{$\begin{array}{c}\text { Ações } \\
\text { Deliberadas }\end{array}$} & $\begin{array}{l}\text { Coef. Correlação } \\
\text { de Pearson }\end{array}$ &,- 027 & ,081 &,- 109 &,- 036 \\
\hline & Valor $p$ & ,689 & ,232 & 109 & ,596 \\
\hline \multirow{2}{*}{ Autodisciplina } & $\begin{array}{l}\text { Coef. Correlação } \\
\text { de Pearson }\end{array}$ &,- 049 &, 022 &,- 076 &,- 054 \\
\hline & Valor $\mathrm{p}$ & ,472 & ,744 & ,262 & ,429 \\
\hline
\end{tabular}




\begin{tabular}{c|c|c|c|c|c}
\hline \multirow{2}{*}{$\begin{array}{c}\text { Hábitos } \\
\text { Saudáveis }\end{array}$} & $\begin{array}{c}\text { Coef. Correlação de } \\
\text { Pearson }\end{array}$ &,- 001 &, 100 &,- 088 &,- 019 \\
\cline { 2 - 5 } & Valor $p$ &, 983 &, 142 &, 197 &, 779 \\
\hline \multirow{2}{*}{ Confiabilidade } & $\begin{array}{c}\text { Coef. Correlação de } \\
\text { Pearson }\end{array}$ & $\mathbf{, 2 7 0}^{* *}$ & $\mathbf{, 4 1 3}^{* *}$ &, 060 &, 110 \\
\cline { 2 - 6 } & Valor $p$ & $\mathbf{, 0 0 0}$ & $\mathbf{, 0 0 0}$ &, 379 &, 106 \\
\hline
\end{tabular}

Fonte: dados da pesquisa.

Nota: ** Correlação significativa a um nível de significância de $1 \%$

* Correlação significativa a um nível de significância de 5\%

\subsection{Análise de Regressão Linear}

A regressão linear múltipla será utilizada para analisar a hipótese H3 (Materialismo está negativamente relacionado à Simplicidade Voluntária, apesar da influência das variáveis moderadoras Autocontrole e LTO). Maroco (2007, p. 638) salienta a importância dos efeitos de moderação (interação) em Ciências do comportamento, pois "fatores específicos podem ser capazes de reduzir ou aumentar quer a magnitude quer a direção do efeito de uma ou mais variáveis preditoras (independentes) na variável de resposta (variável dependente)".

A H3, que relaciona negativamente a Simplicidade Voluntária ao Materialismo apesar da influência das variáveis moderadoras de Autocontrole ou LTO, pode ser verificada a partir dos seus coeficientes de regressão padronizados, assim como dos valores de $\mathrm{p}$ correspondentes, comparando os modelos com e sem variáveis moderadoras.

A tabela 15 permite constatar que indivíduos com maior Centralidade apresentam menor Simplicidade Voluntária. A Felicidade (dimensão do Materialismo) influencia de forma negativa e significativa $(p=0,019)$ a Vida Simples, e o Sucesso influencia de forma positiva e significativa a Ponderação $(p=0,027)$.

O poder de explicação dos modelos é fraco, variando o coeficiente de determinação de 0,019 (para a Autossuficiência, sendo o modelo não significativo, com um valor $p$ de 0,259 na análise de variância) a 0,462 (para a Ponderação), de acordo com a Tabela 11.

Tabela 11 - Regressão Linear do construto Simplicidade Voluntária, sem variáveis moderadoras

\begin{tabular}{|c|c|c|c|c|c|}
\hline $\begin{array}{l}\text { Variável In- } \\
\text { dependente }\end{array}$ & Variável Dependente & SV (geral) & Ponderação & Vida Simples & Autossuficiência \\
\hline \multirow[t]{2}{*}{ Centralidade } & \begin{tabular}{|l} 
Coef. Regressão \\
Padronizado
\end{tabular} & $-0,501 * *$ & $-0,674^{* *}$ & $-0,330 * *$ & $-0,094$ \\
\hline & Valor $\mathrm{p}$ & 0,000 & 0,000 & 0,000 & 0,179 \\
\hline \multirow[t]{2}{*}{ Felicidade } & $\begin{array}{l}\text { Coef. Regressão } \\
\text { Padronizado }\end{array}$ & $-0,090$ & $-0,053$ & $-0,154^{*}$ & 0,004 \\
\hline & Valor $\mathrm{p}$ & 0,142 & 0,317 & 0,019 & 0,953 \\
\hline \multirow{2}{*}{ Sucesso } & $\begin{array}{l}\text { Coef. Regressão } \\
\text { Padronizado }\end{array}$ & $-0,028$ & $0,116 *$ & $-0,090$ & $-0,087$ \\
\hline & Valor $\mathrm{p}$ & 0,645 & 0,027 & 0,164 & 0,216 \\
\hline \multicolumn{2}{|c|}{ Coeficiente de determinação (R2) } & 0,287 & 0,462 & 0,181 & 0,019 \\
\hline \multicolumn{2}{|r|}{ F (ANOVA) } & 28,767 & 61,275 & 15,760 & 1,351 \\
\hline \multicolumn{2}{|r|}{ Valor $p$} & 0,000 & 0,000 & 0,000 & 0,259 \\
\hline
\end{tabular}

Fonte: dados da pesquisa.

Nota: ** Coeficiente significativo a um nível de significância de $1 \%$

* Coeficiente significativo a um nível de significância de 5\% 
Percebe-se, na Tabela 16, que as variáveis referentes às dimensões do Materialismo já não apresentam influências significativas na Simplicidade Voluntária (geral) nem nas suas dimensões. A LTO influencia de forma significativa e positiva a SV.

O SC apresenta influência significativa e negativa na Simplicidade Voluntária geral $(\mathrm{p}=$ $0,002)$ e nas suas dimensões Ponderação $(0,005)$ e Vida Simples $(0,032)$. Os dados demonstram que indivíduos com maior Autocontrole apresentam menor Simplicidade Voluntária.

Conforme a Tabela 12, verifica-se uma interação significativa entre a Centralidade e a Orientação a Longo Prazo nos modelos que têm como variáveis dependentes a SV Geral ( $p=$ $0,010)$, a Vida Simples $(0,022)$ e a Autossuficiência $(0,046)$. Denota-se que a interação da Felicidade com o Autocontrole também é significativa $(p=0,013)$ para prever a Ponderação. Contudo, a Felicidade não apresenta influência significativa na Ponderação nem antes nem depois da inclusão das variáveis moderadoras.

Tabela 12 - Regressão Linear do construto Simplicidade Voluntária, com variáveis moderadoras

\begin{tabular}{|c|c|c|c|c|c|}
\hline $\begin{array}{c}\text { Variável } \\
\text { Independente }\end{array}$ & Variável Dependente & SV (geral) & Ponderação & $\begin{array}{l}\text { Vida } \\
\text { Simples }\end{array}$ & Autossuficiência \\
\hline \multirow{2}{*}{ Centralidade } & $\begin{array}{l}\text { Coef. Regressão } \\
\text { Padronizado }\end{array}$ & $-0,218$ & $-0,507$ & 0,035 & ,003 \\
\hline & Valor $\mathrm{p}$ & ,569 & 128 & ,932 & ,995 \\
\hline \multirow{2}{*}{ Felicidade } & $\begin{array}{l}\text { Coef. Regressão } \\
\text { Padronizado }\end{array}$ & $-0,256$ & $-0,317$ & $-0,541$ & ,264 \\
\hline & Valor $\mathrm{p}$ & ,432 & ,263 & 120 & 499 \\
\hline \multirow{2}{*}{ Sucesso } & $\begin{array}{l}\text { Coef. Regressão } \\
\text { Padronizado }\end{array}$ &,- 051 & 120 & 0,114 &,- 326 \\
\hline & Valor $\mathrm{p}$ & ,903 & ,740 & ,798 &, 517 \\
\hline \multirow[t]{2}{*}{ LTO } & $\begin{array}{l}\text { Coef. Regressão } \\
\text { Padronizado }\end{array}$ & $1,171 * *$ & $0,898 * *$ & ,721* & ,917* \\
\hline & Valor $\mathrm{p}$ & ,000 & ,002 & ,041 & ,021 \\
\hline \multirow{2}{*}{ Autocontrole } & $\begin{array}{l}\text { Coef. Regressão } \\
\text { Padronizado }\end{array}$ & $-1,044 * *$ & $-0,81 * *$ &,$- 748 *$ &,- 713 \\
\hline & Valor $\mathrm{p}$ &, 002 &, 005 & ,032 & , 070 \\
\hline \multirow{2}{*}{$\begin{array}{l}\text { Centralidade* } \\
\text { Autocontrole }\end{array}$} & $\begin{array}{l}\text { Coef. Regressão } \\
\text { Padronizado }\end{array}$ & ,654 & 138 & ,470 & ,800 \\
\hline & Valor $\mathrm{p}$ & 109 & ,695 & 277 & 102 \\
\hline \multirow{2}{*}{$\begin{array}{c}\text { Felicidade* } \\
\text { Autocontrole }\end{array}$} & \begin{tabular}{|l|} 
Coef. Regressão \\
Padronizado \\
\end{tabular} & ,461 & ,891* & 300 &,- 172 \\
\hline & Valor $\mathrm{p}$ & ,260 & ,013 & ,489 & ,725 \\
\hline \multirow{2}{*}{$\begin{array}{c}\text { Sucesso* } \\
\text { Autocontrole }\end{array}$} & $\begin{array}{l}\text { Coef. Regressão } \\
\text { Padronizado }\end{array}$ & ,467 & ,468 & 111 & ,420 \\
\hline & Valor $\mathrm{p}$ & ,251 & ,185 & ,797 & ,390 \\
\hline \multirow{2}{*}{ Centralidade*LTO } & $\begin{array}{l}\text { Coef. Regressão } \\
\text { Padronizado }\end{array}$ & $-1,098 * *$ &,- 349 & $-1,030 *$ & $-1,012^{*}$ \\
\hline & Valor $p$ & ,010 & ,340 & ,022 & ,046 \\
\hline \multirow[t]{2}{*}{ Felicidade* LTO } & $\begin{array}{l}\text { Coef. Regressão } \\
\text { Padronizado }\end{array}$ &,- 315 &,- 652 & 116 &,- 133 \\
\hline & Valor $\mathrm{p}$ & ,448 & ,072 & ,794 & ,790 \\
\hline \multirow{2}{*}{ Sucesso* LTO } & $\begin{array}{l}\text { Coef. Regressão } \\
\text { Padronizado }\end{array}$ &,- 466 &,- 472 &,- 367 &,- 181 \\
\hline & Valor $\mathrm{p}$ & ,228 & 160 & ,372 & ,696 \\
\hline \multicolumn{2}{|c|}{ Coeficiente de determinação (R2) } & ,349 &, 509 & ,263 &, 060 \\
\hline \multicolumn{2}{|c|}{ F (ANOVA) } & 10,018 & 19,413 & 6,676 & 1,196 \\
\hline \multicolumn{2}{|c|}{ Valor $p$} & ,000 & ,000 & ,000 & ,292 \\
\hline
\end{tabular}

Fonte: dados da pesquisa.

Nota: ** Coeficiente significativo a um nível de significância de $1 \%$

* Coeficiente significativo a um nível de significância de 5\% 
Percebe-se que H3 foi parcialmente refutada, pois a LTO teve um efeito moderador significativo na influência da Centralidade na Simplicidade Voluntária, ou seja, teve papel relevante a relação entre o Materialismo e a SV, assim como a interação entre Felicidade e Autocontrole foi significativa para prever a dimensão Ponderação da SV. Esses achados dão luz a uma perspectiva diferente daquela estabelecida por Nepomuceno (2012), que afirmou que a SV é o único estilo de vida essencialmente anticonsumista, motivo pelo qual manteria relação negativa com o materialismo independente da influência de quaisquer variáveis.

No que diz respeito à avaliação da qualidade do ajustamento dos modelos com variáveis moderadoras, a análise de variância indica que, considerando como variável dependente a Simplicidade Voluntária (geral), a Ponderação ou a Vida simples, os modelos ajustados aos dados são significativos. Já o modelo ajustado para a variável dependente Autossuficiência não se revelou significativo. O coeficiente de determinação do modelo para a Autossuficiência apresenta-se muito baixo $(0,06)$, indicando que apenas $6 \%$ da variabilidade desta variável é explicada pelo modelo. 0 modelo com melhor qualidade de ajustamento é o relativo à Ponderação, explicando cerca de $51 \%$ da variabilidade desta variável.

\section{CONSIDERAÇÕES FINAIS}

Esta pesquisa buscou analisar a influência do Materialismo no estilo de vida pautado na Simplicidade Voluntária, relativa ao construto de estilo de vida de Anticonsumo e ao papel moderador do Autocontrole e da Orientação a Longo Prazo nas relações.

Quanto a avaliar o Materialismo e o Estilo de vida de Anticonsumo em estudantes de Administração, afirma-se que há correlação negativa entre Simplicidade Voluntária e Materialismo. Notase, contudo, que a Simplicidade Voluntária contraria a ideia central do Materialismo, que consiste, básica e essencialmente, no apego excessivo a bens e posses e na supervalorização do consumo.

Ao analisar a relação entre Simplicidade Voluntária, Autocontrole e Orientação a Longo Prazo, aferiu-se que a Simplicidade Voluntária não possui relação significante com o Autocontrole ou com a Orientação a Longo. Após a regressão linear, percebeu-se, entretanto, que a Orientação a Longo Prazo teve papel relevante na relação entre o Materialismo e a Simplicidade Voluntária, assim como a interação entre Felicidade e Autocontrole foi significativa para prever a dimensão Ponderação da Simplicidade Voluntária.

Tais achados se mostram relevantes em âmbito acadêmico, social e empresarial, pois permitem estabelecer pontos de partida acerca do entendimento de um indivíduo com um (ou até mais de um) novo perfil de consumidor, que possui uma forma diferente de perceber o mundo e as relações que o permeiam. Com base no estudo aqui desenvolvido, é possível estabelecer novas conjunturas devido à riqueza dos resultados apresentados e validados.

Dentre as limitações encontradas ao longo do processo de desenvolvimento deste estudo, podem-se citar a heterogeneidade da amostra; a utilização das variáveis sociodemográficas apenas em caráter descritivo; e, ainda, a não utilização de outros métodos além daqueles abordados, como a Modelagem em Equações Estruturais, que poderiam trazer novas perspectivas acerca dos apontamentos do estudo.

Espera-se, por fim, que este estudo estimule o surgimento de pesquisas que permitam analisar as complexidades do comportamento de anticonsumo, bem como ampliar e consolidar a discussão sobre o tema na área, seja em meio acadêmico ou empresarial. Ao estudar construtos como Materialismo, Anticonsumo, Autocontrole e Orientação a Longo Prazo, esperou-se contribuir com o conhecimento acerca dessas variáveis, de modo a fornecer base bibliográfica e conceitual para demais trabalhos sobre o tema. 


\section{REFERÊNCIAS}

ALBUQUERQUE, F. et al. Motivações para o ciberativismo anticonsumo em comunidades virtuais antimarca. Rev. Portuguesa e Brasileira de Gestão, Lisboa, v. 9, n. 1-2, jun. 2010. Disponível em <http://www. scielo.gpeari.mctes.pt/scielo.php?script $=$ sci_ arttext\&pid=S1645-44642010000100007\&Ing =pt\&nrm=iso $>$. Acesso em 5 jul. 2013.

ALEXANDER, S. The Voluntary Simplicity Movement: Reimagining the Good Life beyond Consumer Culture. The International Journal of Environmental, Cultural, Economic \& Social Sustainability, v. 7, p. 4-20, 2011.

BANISTER, E. N.; HOGG, M. K. Negative Symbolic Consumption and Consumers' Drive for SelfEsteem: The Case of the Fashion Industry. European Journal of Marketing, v. 38, n. 7, p. 850-868, 2004.

BEARDEN, W. O.; MONEY, B.; NEVINS, J. L. A Measure of Long-Term Orientation: Development and Validation. Journal of the Academy of Marketing Science, v. 34, p. 456-67, 2006.

BELK, R. W. Three Scales to Measure Constructs Related to Materialism: Reliability, Validity, and Relationships to Measures of Happiness. Advances in Consumer Research, Duluth, Minnesota, v. 11, n. 1, p. 291-297, 1984.

BELK, R. W. Materialism: trait aspects of living in the material world. Journal of Consumer Research, v. 12, n. 3, p. 265-280, 1985.

BELK, R. Ineluctable mysteries of possession. Journal of Social Behavior, v. 6, n. 6, p. 17-55, 1991.

CHERRIER, H. Anti-consumption discourses and consumer-resistant identities, Journal of Business Research, v. 62, p. 169-180, 2009.

COLLIS, J.; HUSSEY, R. Pesquisa em Administração: Um guia prático para alunos de graduação e pós-graduação. 2. ed. Porto Alegre: Bookman, 2005.
CRAIG-LEES, M.; HILL, C. Understanding Voluntary Simplifiers. Psychology and Marketing, v. 19, n. 2, p.187-210, 2002.

DINIZ, J. R. Culture Jamming: ativisimo e contra-hegemonia. Caligrama, v. 4, n. 1, 2008. Disponível em: <http://www.eca.usp. br/caligrama/atual.htm>. Acesso em: 21 jun. 2013.

ELGIN, D.; MITCHELL; A. Voluntary Simplicity. The Co-Evolution Quarterly. Sumeer, 1977.

ETZIONI, A. Voluntary simplicity characterization, select psychological implications, and societal consequences. Journal of Economic Psychology, v. 19, p. 619643, 1998.

FOURNIER, S. Consumers and Their Brands: Developing Relationship Theory in Consumer Research. Journal of Consumer Research, v. 24, p. 343-73, 1998.

GARCIA, P. A. O. Escala Brasileira de Valores Materiais - EBVM - Elaboração e validação de uma escala para materialismo como valor de consumo. Tese da Universidade de Brasília, Brasília - DF, 87 pgs, 2009.

GOULD, S. J.; HOUSTON, F. S.; MUNDT, J. Faling to Try to Consume: A Reversal of the Usual Consumer Research Perspective, Advances in Consumer Research, v. 24, p. 211-216, 1997.

GREGG, R.B. The Value of Voluntary Simplicity. Wallingford, PA: Pendle Hill, 1936.

GRISI, C. C. H. Contribuições ao Estudo das Técnicas de Segmentação de Mercado - Uma análise de Dados sobre Apostadores da loteria Federal - Dissertação de Mestrado apresentada à Faculdade de Administração, Economia e Contabilidade da universidade de São Paulo (USP), 1986.

GROHMANN, M. Z.; BATTISTELLA, L. F.; BEURON, T. A.; RISS, L. A.; CARPES, A. M.; LUTZ, C. Relação entre materialismo e estilo de consumo: homens e mulheres com 
comportamento díspare? Contaduría y Administración, v. 57, n. 1, p. 185-214, eneromarzo, 2012.

HAIR JR, J. F; et al. Fundamentos de pesquisa de marketing. Porto Alegre: Bookman, 2010.

$\mathrm{HOCH}$, S.J.; LOEWENSTEIN, G.F. Timeinconsistent preferences and consumer selfcontrol. Journal of Consumer Research, v. 17, n. 4, p. 492-508, 1991.

HOGG, M. K. Anti-Constellations: Exploring the Impact of Negation on Consumption Journal of Marketing Management, v. 14, p. 133-158, 1998.

HOLLENBECK, C. R.; ZINKHAN, G. M. Anti-brand communities, negotiation of brand meaning, and the learning process: The case of WalMart. Consumption Markets \& Culture, v. 13, p. 325-345, 2010.

HOWLETT, E.; KEES, J.; KEMP, E. The Role of SelfRegulation, Future Orientation, and Financial Knowledge in Long-Term Financial Decisions. Journal of Consumer Affairs, 42 (Summer): 223-424, 2008.

IVER, R.; MUNCY, J. Purpose and object of anticonsumption. Journal of Business Research, $v$. 62 , n. 2, p. 160-168, 2009.

IWATA, O. An Evaluation of Consumerism and Lifestyle as Correlates of a Voluntary Simplicity Lifestyle. Social Behavior \& Personality: An International Journal 34(5): 557-556, 2006.

KILBOURNE, W. On the Role of Critical Theory in Moving Toward Voluntary Simplicity. Meaning, Measure and Morality of Materialism, p. 161163, 1992.

KILBOURNE, W.; GRUNHAGEN, M.; FOLEY, J. A cross-cultural examination between materialism and individual values. Journal of Economic Psychology, v. 26, n. 5, p. 624-641, 2005.

KOZINETS, R.; HANDELMAN, J.; LEE, M. Don't read this or, who cares what the hell anti- consumption is, anyways? Consumption Markets \& Culture. v.13, n. 3, p. 225-233, 2010.

MALHOTRA, N. K. Pesquisa de marketing: uma orientação aplicada. Porto Alegre: Bookman, 2011.

MARION, G. Idéologie et dynamique du marketing: quelles responsabilités? Décisions Marketing, v. 31, p. 49-61, 2003.

MATOS, E. B. A gênese da resistência criativa nas idéias de agência de certeau e de habitus de Bourdieu. Anais do XXXV Encontro da ANPAD. 2011, Rio de Janeiro: ANPAD, 2011.

MOGILNER, C.; AAKER, J. The time vs. money effect: Shifting product attitudes and decisions through personal connection. Journal of Consumer Research, v. 36, p. 277-291, 2009.

NEPOMUCENO, M. V. "Anti-consumption": The lifestyles of the disciplined materialists. 2012. 149. f. Tese (Doutorado em Filosofia - Administração) - John Molson School of Business. Concordia University. Montreal, 2012.

POYNOR, C.; HAWS, K. L. Lines in the sand: The role of motivated categorization in the pursuit of self-control goals. Journal of Consumer Research, v. 35, n. 5, p. 772-787, 2009.

RICHINS, M. L. The Material Values Scale: Measurement Properties and Developmentof a Short Form. Journal of Consumer Research, Chicago, v. 31, n. 1, p. 209-219, Jun. 2004.

RICK, S. I.; CRYDER, C. E.; LOEWENSTEIN C. Tightwads and Spendthrifts. Journal of Consumer Research, v. 34, p. 767-82, April 2007.

ROSE, P. Mediators of the association between narcissism and compulsive buying: The roles of materialism and impulse control. Psycology of Addictive Behavirs, v. 21, n. 4, p. 576-581, 2007.

ROTHBAUM, F.; WEISZ, J. R.; SNYDER, S. 
Changing the world and changing the self: A two-process model of perceived control. Journal of Personality and Social Psychology, 42, 1982.

SCHMEICHEL, B. J.; BAUMEISTER, R. F. Selfregulatory strength. In R. F. 2004.

SHAMA, A. Coping with Staglation: Voluntary Simplicity. Journal of Marketing, v. 45, n. 3, p. 120-134, 1981.

SHAW, D.; NEWHOLM, T. Voluntary simplicity and the ethics of consumption. Psychology and Marketing, v. 19, p. 167-185, 2002.

SILVA, T. M. B.; COSTA, A. M.; CARVALHO, J. L. F. Resistência ao Consumo em um Circuito Urbano de Parkour, Comunicação, Mídia e Consumo, v. 8, n. 22, p. 119-146, 2011.

STAMMERJOHAN, C; WEBSTER, C.; Trait and situational antecedents to non-consumption. Advances in Consumer Research, v. 29, n.1, p. 126-132, 2002.

SUAREZ, M.; CHAUVEL, M. A.; CASOTTI, L. Motivações e significados do abandono de categoria: aprendizado a partir da investigação com ex-fumantes e ex-proprietários de automóveis. Cad. EBAPE.BR, v. 10, n. 2, p. 411434, June 2012.

TANGNEY, J.P.; BAUMEISTER, R.F.; BOONE, A.L. High self-control predicts good adjustment, less pathology, better grades, and interpersonal success. Journal of Personality, v. 72, p. 271322, 2004.

WATSON, J. J. The relationship of materialism to spending tendencies, saving, and debt. Journal of Economic Psychology, v. 24, n. 3, p. 723-739, 2003.

WORTHINGTON, R.; WHITTAKER, T. Scale development research: A content analysis and recommendations for best practices. Counseling Psychologist, v. 34, p. 806-838, 2006.
ZAVETOSKI, S. The social-psychological bases of anti-consumption attitude. Psychology and Marketing, v. 19, n. 2, p. 149-172, 2002. 
SER OU NÃO TER, EIS A QUESTÃO: UM ESTUDO SOBRE MATERIALISMO E ESTILO DE VIDA

Rev. AdM. UFSM, SANTA MARIA, V. 7, EdIçÃo ESPECIAL, P. 23-42, NOV. 2014

$-42-$ 